\title{
On Representable Ordered Residuated Semigroups
}

\author{
Szabolcs Mikulás \\ Department of Computer Science and Information Systems \\ Birkbeck College, University of London \\ Malet Street, London WC1E 7HX, UK \\ szabolcs@dcs.bbk.ac.uk
}

\begin{abstract}
We show that the equational theory of representable lattice-ordered residuated semigroups is not finitely axiomatizable. We apply this result to the problem of completeness of substructural logics.

Keywords: residuated semigroups, relation algebras, finite axiomatizability 2000 Mathematics Subject Classifications: 03G15, 06F05
\end{abstract}

\section{Introduction}

Residuated algebras have been extensively investigated in the literature, partly because of their connection to substructural logics. A residuated algebra is representable if it is isomorphic to a family of binary relations and the operations are interpreted as "natural" operations on binary relations - see the precise definition below. An important line of research is to determine precisely which classes of representable residuated algebras have finitely axiomatizable equational or quasiequational theories, since such axiomatizability results yield weak or strong completeness results for substructural logics. Indeed, families of binary relations as semantics for substructural logics have been proposed by various researchers, e.g., for the Lambek calculus (LC) by van Benthem and for relevance logics by Dunn and Maddux. Completeness results of this kind include the completeness of the LC [AM94] and the completeness of the relevance logic with mingle RM [Ma]. We address a similar problem here by looking at the problem of expanding the similarity type of LC with (static) disjunction.

In the remainder of this section we define representable algebras and recall a finite axiomatization of representable lower semilattice-ordered residuated semigroups. In the next section, we look at the possibility of extending the similarity type by including join as well. Finally, we will look at the implication of the main result to substructural logics.

Definition 1.1 A representable relation algebra (an RRA) is $\mathfrak{A}=\left(A,+, \cdot,-, ;,,^{\smile}, 1^{\prime}, 0,1\right)$ such that $A$ consists of subsets of an equivalence relation $W$ and + is union, $\cdot$ is intersection, - is set difference, $0=\emptyset, 1=W$ and

$$
\begin{aligned}
x ; y & =\{(u, v) \in W: \exists w((u, w) \in x \&(w, v) \in y)\} \\
x^{\smile} & =\{(u, v) \in W:(v, u) \in x\} \\
1^{\prime} & =\{(u, v) \in W: u=v\}
\end{aligned}
$$

Let $\tau$ be a set of operations definable in RRA. We denote the class of subreducts of RRA to the signature $\tau$ as $\mathrm{R}(\tau)$.

Note that RRAs are residuated: the following definition defines the right- and left residuals of ;

$$
x \backslash y=-\left(x^{\smile} ;-y\right) \text { and } x / y=-\left(-x ; y^{\smile}\right)
$$


and we can define converse negation as well: $\sim x=-\left(x^{\smile}\right)$. The extension of these operations in an RRA with unit $W$ is as follows:

$$
\begin{aligned}
x \backslash y & =\{(u, v) \in W: \forall w((w, u) \in x \Rightarrow(w, v) \in y)\} \\
x / y & =\{(u, v) \in W: \forall w((v, w) \in y \Rightarrow(u, w) \in x)\} \\
\sim x & =\{(u, v) \in W:(v, u) \notin x\}
\end{aligned}
$$

We recall from [AM94] that representable lower semilattice-ordered residuated semigroups are finitely axiomatizable.

Theorem 1.2 The equational and quasiequational theories of $\mathrm{R}(\cdot,,, \backslash, /)$ are finitely axiomatizable.

Indeed, we showed in [AM94] that the following set of quasiequations axiomatizes the class $\mathrm{R}(\cdot, ;, \backslash, /)$ :

- . is a (lower) semilattice

- ; is associative and monotone w.r.t. ·

$$
\left(x \cdot x^{\prime}\right) ;\left(y \cdot y^{\prime}\right) \leq x ; y
$$

- if $x \leq y$, then $x \backslash y$ behaves similarly to a unit element for ;:

$$
z \leq z ;((x \cdot y) \backslash y) \text { and } z \leq((x \cdot y) \backslash y) ; z
$$

and similarly for /:

$$
z \leq z ;(y /(x \cdot y)) \text { and } z \leq(y /(x \cdot y)) ; z
$$

- \and / are the right and left residuals of ;:

$$
x ; y \leq z \Longleftrightarrow y \leq x \backslash z \Longleftrightarrow x \leq z / y
$$

Pratt [Pr90] observed that the quasiequations 7 can be replaced by the following equations:

$$
\begin{aligned}
& x \backslash\left(y \cdot y^{\prime}\right) \leq x \backslash y \\
& x ;(x \backslash y) \leq y \leq x \backslash(x ; y)
\end{aligned}
$$

and the corresponding equations for $/$. Indeed, assuming $x ; y \leq z$, we have $x \backslash(x ; y)=x \backslash(x ; y \cdot z) \leq$ $x \backslash z$ by 8 , and hence $y \leq x \backslash z$ by the second part of 9 . If we assume $y \leq x \backslash z$, then $x ; y \leq x ;(x \backslash z)$ by monotonicity, and hence $x ; y \leq z$ by the first part of 9 . Since 8 and 9 are easily seen to be valid in $\mathrm{R}(\cdot, ;, \backslash, /)$, we are done.

In the next section, we look at the possibility of extending the similarity type by including join as well.

\section{Main result}

We observed in [AM94] that the quasiequational theory of representable distributive lattice-ordered residuated semigroups is not finitely axiomatizable. We strengthen this result below.

Theorem 2.1 The equational and quasiequational theories of $\mathrm{R}(+, \cdot,, ; \backslash, /)$ are not finitely axiomatizable. The same holds if we expand the similarity type by any set of operations definable in RRA. 
Proof: Maddux defines non-representable, finite, integral, symmetric relation algebras $\mathfrak{A}_{n}$ (for $n \in \omega)$ whose ultraproduct is representable [Ma89]. Andréka shows that already the $\{+, \cdot, ;\}-$ reduct of $\mathfrak{A}_{n}$ is not representable, whence $\tau$-subreducts of RRA such that $\{+, \cdot, ;\} \subseteq \tau$ are not finitely axiomatizable, cf. [An91]. Hence the quasivariety $\mathrm{R}(+, \cdot, ;, \backslash, /)$ is not finitely axiomatizable. Here we show that the non-representability of the $\{+, \cdot, ;, \backslash, /\}$-reduct $\mathfrak{B}_{n}$ of $\mathfrak{A}_{n}$ is witnessed by an equation.

We recall that $\mathfrak{A}_{n}$ has the following atoms: identity $1^{\prime}, q_{i}$ for $1 \leq i \leq m$, and $p_{j}$ for $1 \leq$ $j \leq n$ with $m=3 \cdot n$ !. Composition is defined such that $q_{i+1} \leq p_{1} ; q_{i}$ for every $i<m$ and $0=q_{i} \cdot 1^{\prime} ; q_{j}=q_{r} \cdot q_{s} ; q_{t}=p_{l} \cdot p_{l} ; p_{l}$ for every $1 \leq i \neq j \leq m, 1 \leq r, s, t \leq m$, and $1 \leq l \leq n$. Furthermore, $q_{m} \backslash q_{m}=1^{\prime}$. It follows that a representation of the $\{+, \cdot, ;\}$-reduct of $\mathfrak{A}_{n}$ would require a coloring of the edges of a graph $U$ with $|U| \geq 3 \cdot n$ ! using the colors $p_{i}$ (for $1 \leq i \leq n$ ) but without monochromatic triangles - an impossible task. See Figure 1, where $U=\left\{u_{1}, \ldots, u_{m}\right\}$ and every dotted arrow should have a color $p_{i}$ for some $1 \leq i \leq n$.

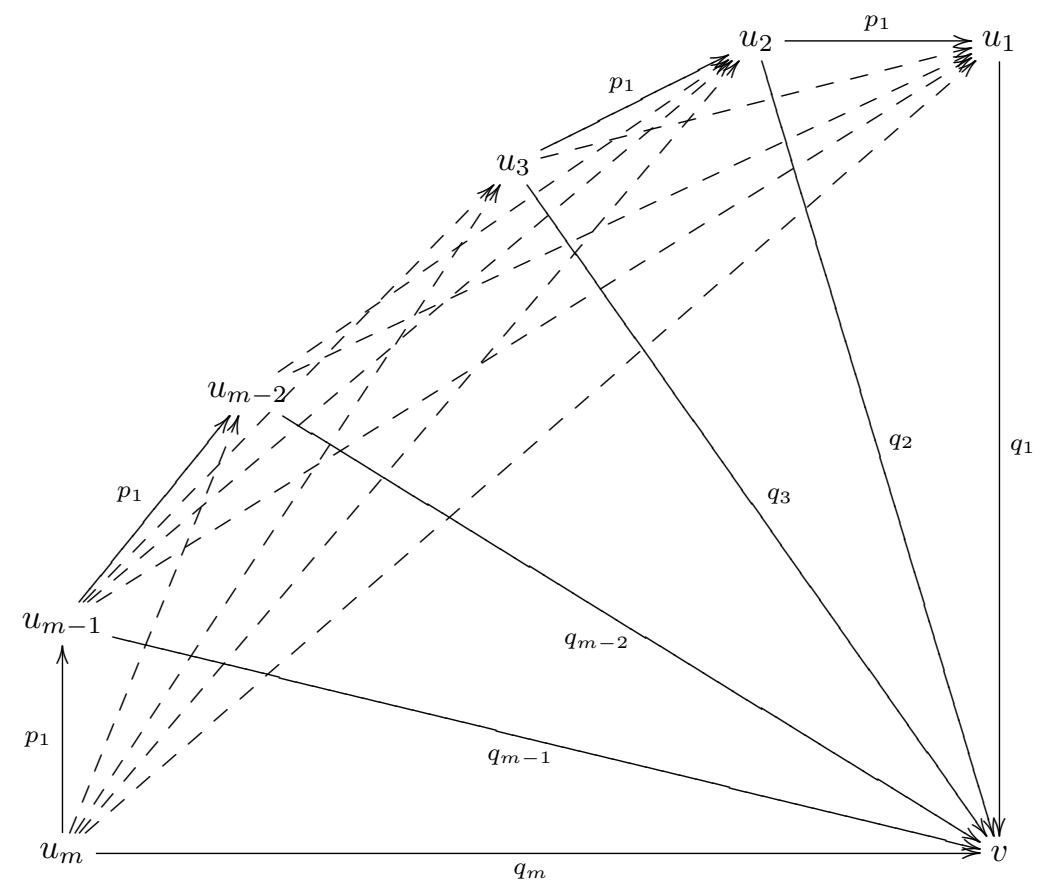

Figure 1: The reason for non-representability

Below we use the abbreviation $x^{i+1}$ that is recursively defined by $x^{i+1}=x ; x^{i}$ with the convention that $x^{0} ; y=y$ for every term $y$. Let $x_{i}, y_{j}$ and $z_{0}$ be distinct variables for $1 \leq i \leq n$ and $1 \leq j \leq m$ and let $Z$ stand for $\sum\left\{x_{i}: 1 \leq i \leq n\right\}+\sum\left\{y_{j}: 1 \leq i \leq m\right\}+\left(y_{m} \backslash y_{m}\right)$. We define the equation $e_{n}$ for $n \in \omega$ as

$$
\sigma_{n} ;\left(\alpha_{n} \cdot \beta_{n} \cdot \gamma_{n} \cdot \delta_{n}\right) \leq z_{0}
$$

where

$$
\begin{aligned}
\sigma_{n} & =y_{m} \cdot\left(x_{1} ;\left(y_{m-1} \cdot\left(\left(x_{1} \cdot x_{1} \backslash Z\right) ;\left(y_{m-2} \cdot\left(x_{1} \cdot x_{1} \backslash Z \cdot\left(x_{1} ; x_{1}\right) \backslash Z\right) ;(\ldots)\right)\right)\right)\right) \\
\alpha_{n} & =\prod\left\{\left(x_{1}^{m-i} ;\left(y_{i} \cdot y_{j}\right)\right) \backslash z_{0}: 1 \leq i \neq j \leq m\right\} \\
\beta_{n} & =\prod\left\{\left(x_{1}^{i} ;\left(x_{l} \cdot x_{l} ; x_{l}\right) ; y_{j}\right) \backslash z_{0}: 1 \leq i, j \leq m, 1 \leq l \leq n\right\} \\
\gamma_{n} & =\prod\left\{\left(x_{1}^{i} ;\left(y_{m-i} \cdot y_{m} \backslash y_{m} ; y_{m-j}\right)\right) \backslash z_{0}: 0<i<j<m\right\} \\
\delta_{n} & =\prod\left\{\left(x_{1}^{i} ;\left(y_{m-i} \cdot y_{k} ; y_{j}\right)\right) \backslash z_{0}: 1 \leq i<m, 1 \leq j, k \leq m\right\}
\end{aligned}
$$


We claim that $e_{n}$

1. fails in $\mathfrak{B}_{n}$

2. is valid in representable algebras.

For item 1 , let $\iota$ be the evaluation of the variables

$$
\iota\left(x_{i}\right)=p_{i} \quad \iota\left(y_{j}\right)=q_{j} \quad \iota\left(z_{0}\right)=0
$$

Then $\iota\left(R H S\left(e_{n}\right)\right)=\iota\left(z_{0}\right)=0$. On the other hand, $\iota\left(L H S\left(e_{n}\right)\right) \neq 0$ because of the following. First note that $\iota(Z)=1$, since $\iota\left(y_{m} \backslash y_{m}\right)=q_{m} \backslash q_{m}=1^{\prime}$. Then we have $\iota(\tau \backslash Z)=1$ for any term $\tau$. In $\mathfrak{B}_{n}, q_{i+1} \leq p_{1} ; q_{i}$ for every $1 \leq i<m$, whence $\iota\left(\sigma_{n}\right) \neq 0$. Furthermore, the terms on the left of $\backslash$ in $\alpha_{n}, \beta_{n}, \gamma_{n}$ and $\delta_{n}$ evaluate to 0 , since the meet of distinct atoms is 0 (for $\alpha_{n}$ ), there are no monochromatic triangles for $p_{i}$ (for $\left.\beta_{n}\right), q_{m} \backslash q_{m} \leq 1^{\prime}$ (for $\gamma_{n}$ ) and there are no $q$-triangles (for $\left.\delta_{n}\right)$. Hence $\iota\left(\alpha_{n}\right)=\iota\left(\beta_{n}\right)=\iota\left(\gamma_{n}\right)=\iota\left(\delta_{n}\right)=1$. Thus $\iota\left(\operatorname{LHS}\left(e_{n}\right)\right) \neq 0$.

For item 2 , let $\mathfrak{C}=(C,+, \cdot, ;, \backslash, /)$ be a representable algebra and $\iota$ be an arbitrary valuation of the variables. Assume that $\left(u_{m}, u\right) \in \iota\left(L H S\left(e_{n}\right)\right)$. Then there is $v$ such that $\left(u_{m}, v\right) \in \iota\left(\sigma_{n}\right)$ and $(v, u) \in \iota\left(\alpha_{n} \cdot \beta_{n} \cdot \gamma_{n} \cdot \delta_{n}\right)$. By $\left(u_{m}, v\right) \in \iota\left(\sigma_{n}\right)$, we have $\left(u_{i+1}, u_{i}\right) \in \iota\left(x_{1}\right)$ and $\left(u_{i}, v\right) \in \iota\left(y_{i}\right)$ for every $1 \leq i<m$. See Figure 2 .

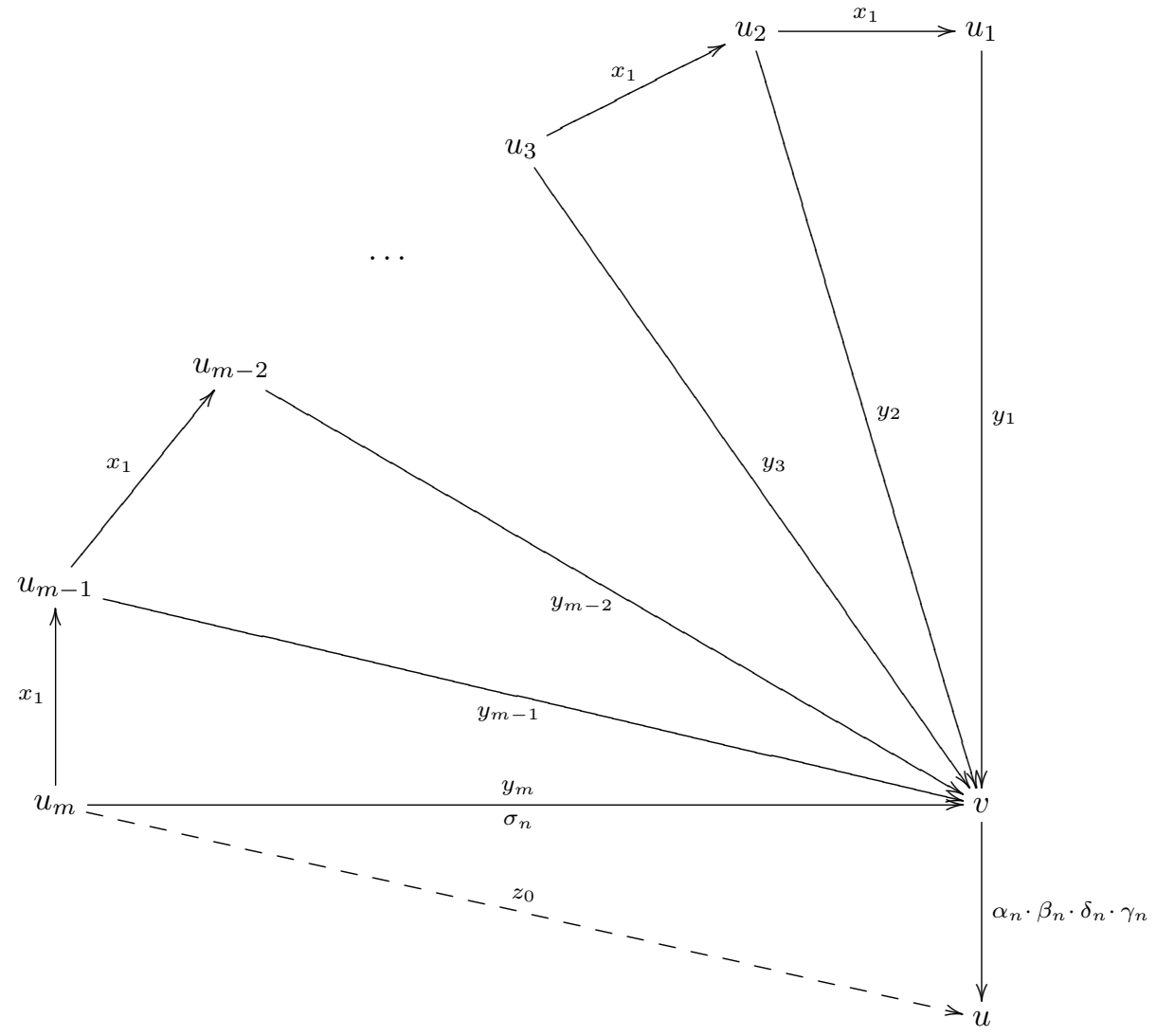

Figure 2: The validity of $e_{n}$

First we consider the case where there are $i \neq j$ such that $u_{i}=u_{j}$. In this case, we have $\left(u_{i}, v\right) \in \iota\left(y_{i}\right) \cdot \iota\left(y_{j}\right)$ and thus $\left(u_{m}, v\right) \in \iota\left(x_{1}^{m-i} ;\left(y_{i} \cdot y_{j}\right)\right)$. Since $(v, u) \in \iota\left(\alpha_{n}\right)$, we get $\left(u_{m}, u\right) \in$ $\iota\left(z_{0}\right)=\iota\left(R H S\left(e_{n}\right)\right)$.

Now let us assume that all the $u_{i}$ s are different. Note that

$$
\left(u_{i}, u_{j}\right) \in \iota(Z)=\iota\left(\sum\left\{x_{k}: 1 \leq k \leq n\right\}+\sum\left\{y_{l}: 1 \leq l \leq m\right\}+\left(y_{m} \backslash y_{m}\right)\right)
$$


for every $1 \leq i \neq j \leq m$, by $\left(u_{m}, v\right) \in \iota\left(\sigma_{n}\right)$. If there are $i<j$ and $k$ such that $\left(u_{i}, u_{j}\right) \in \iota\left(y_{k}\right)$, then $\left(u_{m}, v\right) \in \iota\left(x_{1}^{i} ;\left(y_{m-i} \cdot y_{k} ; y_{j}\right)\right)$. Hence $\left(u_{m}, u\right) \in \iota\left(z_{0}\right)$ by $(v, u) \in \iota\left(\delta_{n}\right)$, i.e., $\left(u_{m}, u\right) \in \iota\left(R H S\left(e_{n}\right)\right)$. If there are $i<j$ such that $\left(u_{i}, u_{j}\right) \in \iota\left(y_{m} \backslash y_{m}\right)$, then $\left(u_{m}, v\right) \in \iota\left(\left(x_{1}^{i} ;\left(y_{m-i} \cdot y_{m} \backslash y_{m} ; y_{m-j}\right)\right)\right)$. Hence $\left(u_{m}, u\right) \in \iota\left(z_{0}\right)$ by $(v, u) \in \iota\left(\gamma_{n}\right)$, i.e., $\left(u_{m}, u\right) \in \iota\left(R H S\left(e_{n}\right)\right)$. It remains to consider the case where, for all $i<j$, there is $l$ such that $\left(u_{i}, u_{j}\right) \in \iota\left(x_{l}\right)$. By $m=3 \cdot n$ !, we have that for some $1 \leq i<j<k \leq m,\left(u_{i}, u_{j}, u_{k}\right)$ is a monochromatic triangle the edges of which are colored with some $\iota\left(x_{l}\right)$. Hence $\left(u_{m}, v\right) \in \iota\left(x_{1}^{m-i} ;\left(x_{l} \cdot x_{l} ; x_{l}\right) ; y_{k}\right)$. Then $\left(u_{m}, v\right) \in \iota\left(z_{0}\right)$ for some $1 \leq i \leq m$, by $(v, u) \in \iota\left(\beta_{n}\right)$. Hence $\left(u_{m}, u\right) \in \iota\left(R H S\left(e_{n}\right)\right)$ in this case as well. Thus $e_{n}$ is indeed valid in representable algebras.

\section{Substructural logics}

In this section, we explain the connection of the main result to substructural logics.

Lambek calculus In [AM94], we showed that the Lambek calculus with static conjunction is complete w.r.t. relational semantics Rel(LC) consisting of binary relations, where fusion $(\bullet$ in the original formulation and ; in our notation) is interpreted as relation composition, conjunction $(\wedge$ or · ) as intersection and $\backslash$ and / as the two residuals of composition. There is one subtle difference between this semantics $\operatorname{Rel}(\mathrm{LC})$ and the subreduct $\mathrm{R}(\cdot, ;, \backslash, /)$ of RRA. Namely, in Rel(LC), we do not require that the union $W$ of all relations is an equivalence relation. In fact, in the completeness proof, $W$ is irreflexive $((x, x) \notin W)$ and antisymmetric (for $x \neq y,(x, y) \in W$ implies $(y, x) \notin W$ ), and the residuals are defined as in 1 and 2 with $W$ as a parameter. Since $W$ is antisymmetric, so are $x \backslash y$ and $x / y$, hence 5 and 6 fail in accordance with the absence of the corresponding rules in the original LC [La58]. Thus Theorem 2.1 does not seem to answer the problem of the weak completeness of the LC extended with disjunction. But we claim that essentially the same proof yields the following.

Corollary 3.1 No extension with finitely many axioms of the Lambek calculus with disjunction is weakly complete w.r.t. relational semantics Rel.

Proof: In this case a representable algebra has the form $\mathfrak{D}=(D,+, \cdot, ;, \backslash, /)$ where $D$ is a collection of binary relations, + is union, $\cdot$ is intersection, ; is relation composition as before and

$$
\begin{aligned}
& x \backslash y=\{(u, v) \in W: \forall w((w, u) \in x \Rightarrow(w, v) \in y)\} \\
& x / y=\{(u, v) \in W: \forall w((v, w) \in y \Rightarrow(u, w) \in x)\}
\end{aligned}
$$

where $W=\bigcup\{d: d \in D\}$, i.e., $W$ is not necessarily an equivalence relation. An inspection of the proof of Theorem 2.1 reveals that we did not use anywhere that the unit of the representable algebra would be an equivalence relation, and that, in particular, $\iota\left(y_{m} \backslash y_{m}\right)$ would be reflexive ${ }^{1}$. Hence the same argument as above shows that $\mathfrak{B}_{n}$ is not representable while a non-trivial ultraproduct of $\mathfrak{B}_{n}$ is representable ${ }^{2}$, and that the equation $e_{n}$ fails in $\mathfrak{B}_{n}$ while it is valid in representable algebras.

Relevance logic Finally, let us mention an open problem related to relevance logic [AB75, ABD92]. Recall that relevance logics can be soundly interpreted over families of binary relations. Let $\mathrm{R}^{c d}(;, \cdot,+, \backslash, \sim)$ be that subclass of $\mathrm{R}(;, \cdot,+, \backslash, \sim)$ where each algebra is commutative $x ; y=y ; x$ and dense (or square-increasing) $x \leq x ; x$. Let $\mathfrak{A} \in \mathrm{R}^{c d}(;, \cdot,+, \backslash, \sim)$ with unit $W$ and $v$ be a valuation of the propositional atoms into $A$. We extend $v$ to compound formulas by interpreting

\footnotetext{
${ }^{1}$ We used transitivity of the unit, but that holds for $W$ in this case as well because of composition, and $x \backslash x$ always contains the reflexive edges from $W$.

${ }^{2}$ Hence strong completeness fails as well [AM94].
} 
conjunction as intersection, disjunction as union, implication as the residual operation (right and left residuals coincide by commutativity) and relevant negation as converse negation. We define

$$
\mathfrak{A}=\varphi \Longleftrightarrow \mathrm{Id} \subseteq v(\varphi)
$$

where $\mathrm{Id}=\{(u, v) \in W: u=v\}$. Then relevance logic $R$ is sound w.r.t. $\mathrm{R}^{c d}(;, \cdot,+, \backslash, \sim)$, and relevance logic $\mathrm{RM}$ with the mingle axiom is complete w.r.t. the semantics $\mathrm{R}^{c d t}(;, \cdot,+, \backslash, \sim)$, that subclass of $\mathrm{R}^{c d}(;, \cdot,+, \backslash, \sim)$ where each algebra is transitive (or square-decreasing) $x ; x \leq x$, see [Ma]. On the other hand, completeness fails without the mingle axiom: the logic of $\mathrm{R}^{c d}(;, \cdot,+, \backslash, \sim)$ is not finitely axiomatizable [Mi09].

The question is whether we could achieve more in the absence of relevant negation. Let $R^{p}$ denote the fragment of the relevance logic $R$ to the language of fusion, conjunction, disjunction and implication axiomatized by the axioms $A 1-A 11, A 14$ and $A 15$ and derivation rules $R 1, R 2$ of [RM73]. Obviously, $R^{p}$ is sound w.r.t. the semantics defined by the commutative and dense algebras $\mathrm{R}^{c d}(;, \cdot,+, \backslash)$.

The algebras $\mathfrak{A}_{n}$ in the proof of Theorem 2.1 are commutative, but they are not dense, since there are no monochromatic triangles. And this latter fact seems to be crucial in the non-finite axiomatizability proof. Hence we ask the following.

Problem 3.2 Is $R^{p}$ complete w.r.t. the semantics $\mathrm{R}^{c d}(;, \cdot,+, \backslash)$ ? Is the equational theory of $\mathrm{R}^{c d}(;, \cdot,+, \backslash)$ finitely axiomatizable?

We note that the quasiequational theory of $\mathrm{R}^{c d}(;, \cdot,+, \backslash)$ is not finitely axiomatizable, since the $\{;, \cdot,+, \backslash\}$-reduct of the algebras $\mathfrak{A}_{n}$ from [Mi09] are not representable (while their ultraproduct is).

\section{References}

[AB75] A.R. Anderson \& N.D. Belnap, Entailment. The Logic of Relevance and Necessity. Vol. I. Princeton University Press. 1975.

[ABD92] A.R. Anderson, N.D. Belnap \& J.M. Dunn, Entailment. The Logic of Relevance and Necessity. Vol. II. Princeton University Press. 1992.

[An91] H. AnDRÉKA, "Representation of distributive lattice-ordered semigroups with binary relations", Algebra Universalis, 28:12-25, 1991.

[AM94] H. ANdrÉKA \& Sz. Mikulás, "Lambek calculus and its relational semantics: completeness and incompleteness", Journal of Logic, Language and Information, 3:1-37, 1994.

[La58] J. LAmbeK, "The mathematics of sentence structure" American Mathematical Monthly, $65: 154-170,1958$.

[Ma89] R.D. MAdDux, "Non-finite-axiomatizability results for cylindric and relation algebras", Journal of Symbolic Logic, 54(3):951-974, 1989.

[Ma] R.D. Maddux, "Relevance logic and the calculus of relations" Review of Symbolic Logic, to appear.

[Mi09] Sz. Mikulás, "Algebras of relations and relevance logic", Journal of Logic and Computation, 19:305-321, 2009.

[Pr90] V. Prate, "Action logic and pure induction", In J. van Eijck, editor, Logics in AI: European Workshop JELIA '90, pages 97-120. Springer-Verlag, 1990.

[RM73] R. Routley \& R.K. Meyer, "The semantics of entailment (I)", in H. Leblanc (ed.), Truth, Syntax and Modality, pages 199-243. North-Holland 1973. 